\title{
runBioSimulations: an extensible web application that simulates a wide range of computational modeling frameworks, algorithms, and formats
}

\author{
Bilal Shaikh ${ }^{\circledast 1}$, Gnaneswara Marupilla², Mike Wilson ${ }^{\circledR 2}$, Michael L. Blinov ${ }^{\circledR 2}$, Ion I. \\ Moraru ${ }^{\oplus{ }^{*}}$ and Jonathan R. Karr ${ }^{\oplus 1, *}$
}

${ }^{1}$ Icahn Institute for Data Science and Department of Genetics and Genomic Sciences, Icahn School of Medicine at
Mount Sinai, 12555 th Avenue, Suite C2, New York, NY 10029, USA and ${ }^{2}$ Center for Cell Analysis and Modeling,
University of Connecticut School of Medicine, 263 Farmington Avenue, Farmington, CT 06030, USA.

\begin{abstract}
2 Comprehensive, predictive computational models ${ }_{3}$ have significant potential for science, bioengineer${ }_{4}$ ing, and medicine. One promising way to achieve more predictive models is to combine submodels of ${ }_{6}$ multiple subsystems. To capture the multiple scales 7 of biology, these submodels will likely require multi\& ple modeling frameworks and simulation algorithms. 9 Several community resources are already available 10 for working with many of these frameworks and 1 algorithms. However, the variety and sheer number 2 of these resources make it challenging to find and ${ }_{3}$ use appropriate tools for each model, especially ${ }_{4}$ for novice modelers and experimentalists. To make ${ }_{5}$ these resources easier to use, we developed ${ }_{16}$ runBioSimulations (https://run.biosimulations.org), 7 a single web application for executing a broad range ${ }_{18}$ of models. runBioSimulations leverages community 19 resources, including BioSimulators, a new open registry of simulation tools. These resources cur21 rently enable runBioSimulations to execute nine 22 frameworks and 44 algorithms, and they make runBioSimulations extensible to additional frameworks ${ }_{24}$ and algorithms. runBioSimulations also provides ${ }_{25}$ features for sharing simulations and interactively ${ }_{26}$ visualizing their results. We anticipate that runBioSimulations will foster reproducibility, stimulate col${ }_{28}$ laboration, and ultimately facilitate the creation of more predictive models.
\end{abstract}

\section{${ }_{30}$ INTRODUCTION}

${ }_{31}$ More comprehensive and predictive models have significant 32 potential for biology, bioengineering, and medicine. For ${ }_{33}$ example, models of entire cells could help synthetic biologists ${ }_{34}$ design cells and help physicians personalize medicine $(1,2)$.

35 Due to the complexity of biology, building such models ${ }_{36}$ will likely require collaboration among many modelers and ${ }_{37}$ experimentalists. One promising way to harness this diverse
38 expertise is to combine multiple submodels of individual 39 subsystems, each developed by a small group of experts.

40 To capture the multiple scales relevant to biology at a ${ }_{41}$ practical computational cost, these submodels will likely 42 require multiple modeling frameworks. For example, a model 43 of the phenotypic heterogeneity of single cells might need 44 to capture slow processes, such as transcription, precisely, 45 while fast processes, such as metabolism, could be captured 46 coarsely. Numerous frameworks and simulation algorithms 47 already exist for several scales. For example, stochastic kinetic 48 simulations can capture the cell-to-cell variability in gene 49 expression, and flux-balance analysis (FBA) can capture the 50 distribution of fluxes over metabolic networks.

To facilitate collaboration, several model formats have been 52 created for these frameworks and algorithms. For example, the ${ }_{53}$ BioNetGen Language (BNGL, 3), CellML (4), NeuroML (5), ${ }_{54}$ and the Systems Biology Markup Language (SBML, 6) can 55 capture kinetic models, and the SBML flux balance constraints 56 (SBML-fbc; 7) and qualitative modeling (SBML-qual; 8) 57 packages can capture flux balance and logical models.

${ }_{58}$ Furthermore, numerous software tools support these for59 mats. For example, BioNetGen (9), NFSim (10), and Virtual ${ }_{60}$ Cell (VCell; 11) support BNGL; BoolNet (12) and The Cell ${ }_{61}$ Collective (13) support SBML-qual; CBMPy (14), COBRApy 62 (15), and The Cell Collective support SBML-fbc; COPASI ${ }_{63}$ (16), JWS Online (17), StochSS (18), tellurium (19), and ${ }_{64}$ VCell (11) support SBML; OpenCOR supports CellML (20); 65 and Open Source Brain supports NeuroML (21).

These resources provide experts rich silos for modeling ${ }_{67}$ individual subsystems. However, this siloing poses obstacles 68 to composing models of multiple subsystems and scales ${ }_{69}$ into comprehensive models. The effort required to learn the 70 unique frameworks, algorithms, formats, software tools, and 71 conventions associated with each silo also impedes collabora72 tion, especially for novice modelers and experimentalists.

To help investigators use these resources, we developed 74 runBioSimulations, an extensible REST API and graphical 75 web application for executing simulations involving a broad 76 range of frameworks, algorithms, and model formats. runBio77 Simulations leverages several community resources, including 78 model formats such as SBML, the Simulation Experiment 79 Description Language (SED-ML, 22), the COMBINE archive

\footnotetext{
*To whom correspondence should be addressed. Tel: +1 212-824-9664 (JRK), +1 860-679-2908 (IIM); Email: karr@mssm.edu, moraru@uchc.edu
} 


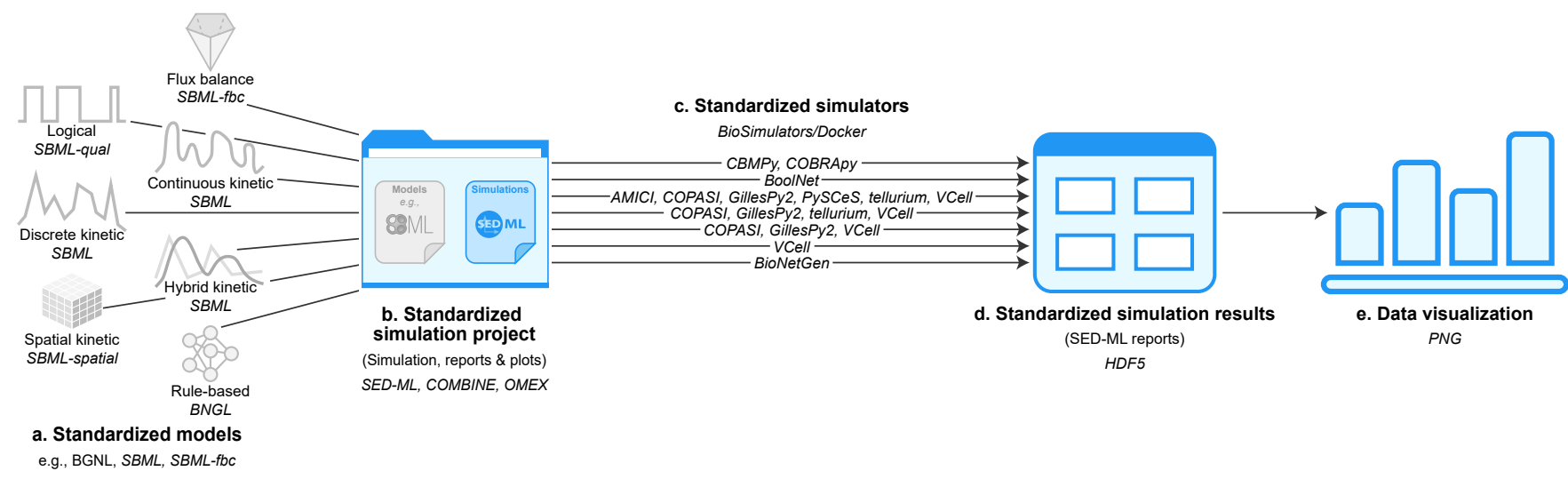

Figure 1. runBioSimulations is an extensible, standards-driven web application for executing models across a broad range of modeling frameworks, simulation algorithms, and model formats. runBioSimulations can execute models (a) and simulations (b) described with community resources such as COMBINE, SBML, and SED-ML with standardized simulation tools registered with BioSimulators (c). runBioSimulations produces results in HDF5 format (d). runBioSimulations also provides tools for interactively visualizing simulation results (e).

80 format (23), and BioSimulators (https://biosimulators.org), 81 a new open registry of standardized simulation tools. As 82 of this writing, runBioSimulations supports nine frame83 works, 44 algorithms, and five model formats (Table S1). 84 This includes 37 algorithms for continuous, discrete, hybrid, 85 and rule-based kinetic simulation with BNGL and SBML; ${ }_{86}$ five algorithms for flux balance simulation with SBML-fbc; 87 and three algorithms for logical simulation with SBML88 qual. Importantly, the community can expand runBioSim89 ulations to additional frameworks, algorithms, and formats 90 by contributing additional simulation tools to BioSimula91 tors. runBioSimulations also provides features for visualizing 92 simulation results and debugging, managing, and sharing sim93 ulations. Furthermore, the runBioSimulations API enables the 94 community to develop additional front-end applications that 95 utilize runBioSimulations' unique simulation capabilities. For 96 example, model repositories could use the API to provide 97 interactive simulations of their models.

By making it easier to execute a broad range of mod${ }_{99}$ els, we anticipate that runBioSimulations will foster model 100 reuse, bolster collaboration, and empower peer review. In turn, 101 we anticipate this will accelerate the development of more 102 comprehensive and more predictive models.

103 Below, we describe the key features of runBioSimulations, 104 its architecture, and how it facilitates model reuse and collab105 oration. In addition, we outline our future plans for runBio106 Simulations. The Supplementary Data summarizes the frame107 works, algorithms, formats, and simulation tools supported 108 by runBioSimulations; provides additional information about 109 the implementation of runBioSimulations; presents a case 110 study of using runBioSimulations to evaluate the practical 111 reusability of existing published simulations to individual 112 investigators that illustrates the utility of runBioSimulations; 113 compares runBioSimulations to other tools; and outlines how 114 the community can contribute to runBioSimulations.

\section{KEY FEATURES}

116 The key feature of runBioSimulations is the capability to 117 execute a broad range of simulations that involve a variety 118 of modeling frameworks, simulation algorithms, and model
119 formats from a single, simple, consistent interface. This 120 is achieved through a modular architecture that leverages ${ }_{121}$ existing resources including model formats such as SBML, 122 SED-ML, and the COMBINE archive format to encapsulate 123 the details of each framework, algorithm, and model for124 mat. This architecture is implemented as a REST API. The 125 runBioSimulations graphical user interface (GUI) provides 126 investigators a user-friendly client to this powerful API.

${ }_{127}$ The starting point to using runBioSimulations is a ${ }_{128}$ COMBINE archive that contains one or more models in a for129 mat such as BNGL or SBML and describes one or more simu130 lations of these models in SED-ML. Users can obtain models 131 and simulations encoded in these formats from repositories ${ }_{132}$ such as BioModels (24) or use tools such as VCell to create ${ }_{133}$ models and simulations in these formats. Models and simu${ }_{134}$ lations can be packaged into COMBINE archives using tools 135 such as CombineArchiveWeb (25).

${ }_{136}$ The runBioSimulations GUI enables users to execute mod$137 \mathrm{els}$ and retrieve and visualize their results in three simple 138 steps. First, users use the GUI to select a COMBINE 139 archive to execute and a simulation tool to run the archive 140 (Figure 2b). Users can choose any of the standardized sim${ }_{141}$ ulation tools available in the BioSimulators registry and any 142 of their versions. To help investigators find tools that are ${ }_{143}$ compatible with specific types of models and/or that sup144 port specific simulation algorithms, BioSimulators provides 145 detailed information about the capabilities of each simula146 tion tool (Figure 2a). The ability to use multiple simulators 147 has several benefits. (a) This makes it easier to reuse mod148 els, including older models that require legacy formats. (b) 149 This design makes the simulation logic of runBioSimula150 tions transparent and portable, ensuring users that they can 151 continue work initiated with runBioSimulations onto their 152 own computers using the same simulators, further lowering 153 the barrier to model reuse. (c) Because BioSimulators is an ${ }_{154}$ open registry, this design enables the community to extend the 155 simulation capabilities of runBioSimulations.

${ }_{156}$ Users can manage their simulations and monitor their 157 progress using a table that summarizes their simulations ${ }_{158}$ (Figure 2g). Optionally, users can also provide an address to 
bioRxiv preprint doi: https://doi.org/10.1101/2021.03.05.433787; this version posted March 5, 2021. The copyright holder for this preprint (which was not certified by peer review) is the author/funder, who has granted bioRxiv a license to display the preprint in perpetuity. It is made available under aCC-BY 4.0 International license.

\begin{tabular}{|c|c|c|c|c|c|c|}
\hline a Name & Frameworks & Algorithms & Model formats & Curation & Run & Docs \\
\hline $\mathrm{AMICl}$ & non-spatial continuous & CVODES, IDA & SBML & $\star \star \star \star \star$ & t) & a \\
\hline BioNetGen & $\begin{array}{l}\text { non-spatial continuous, } \\
\text { non-spatial discrete }\end{array}$ & $\begin{array}{l}\text { CVODE, Gillespie direct } \\
\text { algorithm, NFSim agent.. }\end{array}$ & BNGL & $\star \star \star \star \star$ & 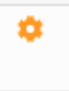 & a \\
\hline BioUML & $\begin{array}{l}\text { flux balance, non-spatial } \\
\text { continuous, non-spatial.. }\end{array}$ & $\begin{array}{l}\text { Dormand-Prince } \\
\text { method, Euler forward. }\end{array}$ & SBML & 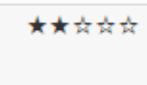 & & a \\
\hline
\end{tabular}
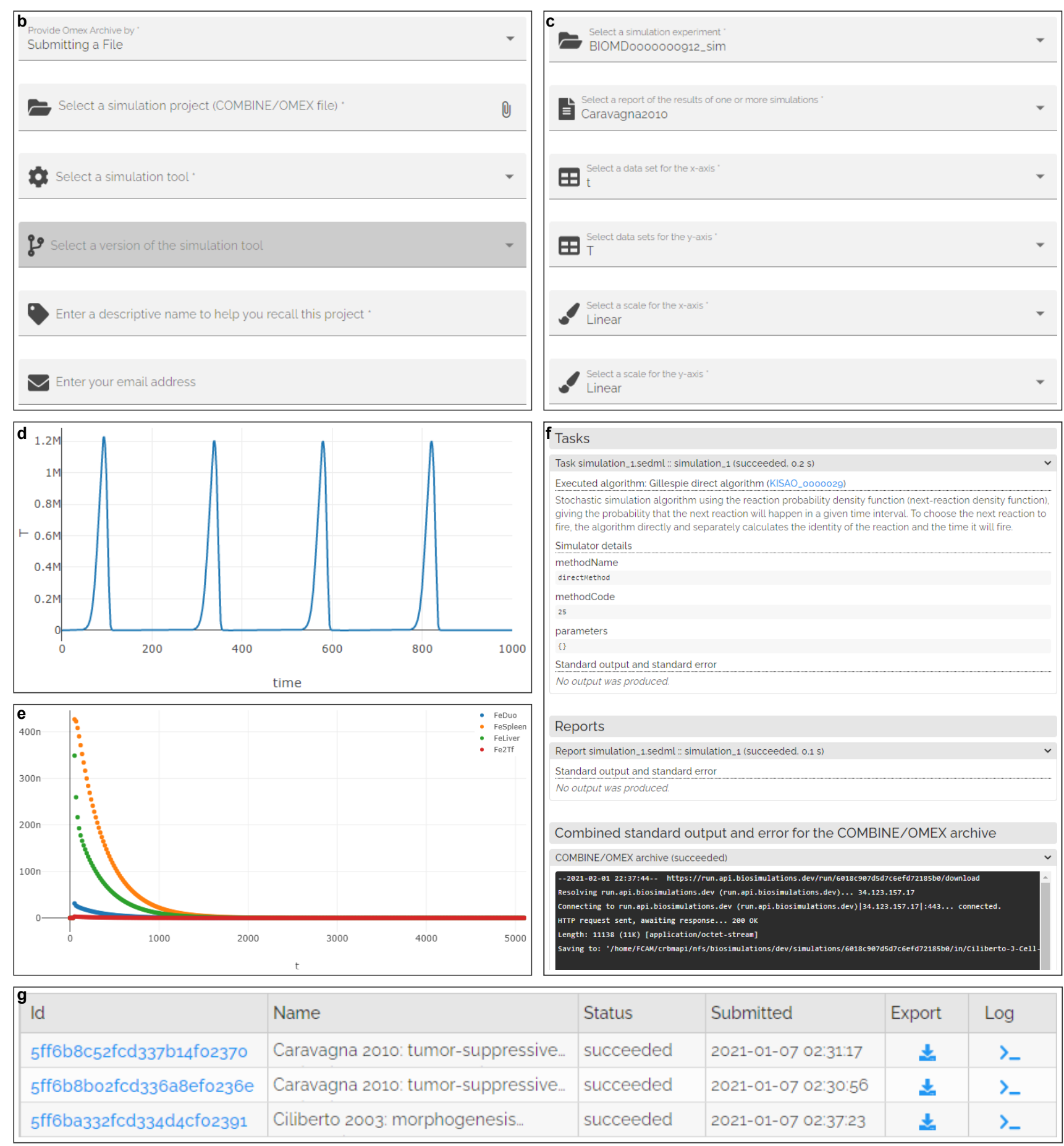

Figure 2. runBioSimulations provides a single GUI for executing a broad range of models and visualizing their results. (a) Users can use BioSimulators to select tools for executing specific simulations. (b-e) runBioSimulations provides simple forms for executing simulations and designing visualizations of their results. runBioSimulations also provides a summary table (g) and structured logs (f) for managing and debugging simulations. 
159 receive emails about the completion of the execution of their 160 archives. This feature is valuable for long simulations.

161 Once simulations complete, users can download and 162 visualize their results. Simulation results can be downloaded ${ }_{163}$ in HDF5 format. The GUI provides users a simple form 164 (Figure 2c) for designing two-dimensional plots of model 165 predictions (Figure 2d, e).

${ }_{166}$ Users also have the option to upload Vega visualizations 167 (26) to visualize simulation results. This enables investi168 gators to visualize their simulation results with a broad 169 range of charts, as well as custom, interactive, publication170 quality diagrams. This also makes it easier to reuse 171 visualizations across multiple simulation conditions by re172 painting them with results of alternative simulations. Together, 173 this combination of runBioSimulations and Vega ensures that 174 the provenance of simulation results and visualizations of 175 simulation results are transparent by capturing all of the 176 information needed to reproduce each result and visualization, 177 including the model, simulation, and simulator which 178 generated each result and the transformations used to map 179 each result to each diagram.

180 To help users debug simulations, the GUI also displays 181 structured logs of their execution (Figure 2f). This can help 182 direct users to errors in specific SED-ML tasks and outputs.

183 runBioSimulations also makes it easy for users to share 184 simulations and their results via persistent URLs similar 185 to file sharing services such as Google Drive. These links 186 enable users to revisit their simulation results, share simula${ }_{187}$ tions with collaborators, anonymously share simulations with 188 peer reviewers, and publish simulations by embedding links 189 into articles. runBioSimulations is particularly well-suited 190 to sharing computationally-expensive simulations because it 191 enables investigators to quickly retrieve their results without 192 having to wait for long simulations to complete.

193 Furthermore, developers can use runBioSimulations' REST 194 API to build additional client applications that leverage run195 BioSimulations' simulation logic. For example, developers 196 could use the API to build additional clients for executing 197 simulations such as Jupyter notebooks or desktop applications.

\section{METHODS}

199 runBioSimulations is composed of a GUI for submitting sim200 ulations, managing simulations, and visualizing their results; 201 services for executing simulations on a high-performance 202 computing (HPC) cluster, monitoring their progress, and ${ }_{203}$ collecting their results; and a database for storing simula204 tions and their results (Figure 3). More information about 205 the design, implementation, and deployment of runBioSimu206 lations is available in the Supplementary Data.

\section{${ }_{207}$ USE CASES}

\section{Publishing simulations}

209 We believe that runBioSimulations' standards-driven design 210 and unique capability to execute a broad range of simulations 211 is ideal for publishing simulations. As more tools embrace 212 SED-ML, runBioSimulations will help authors publish sim213 ulations that other investigators can easily reuse. For exam214 ple, investigators could use runBioSimulations to explore

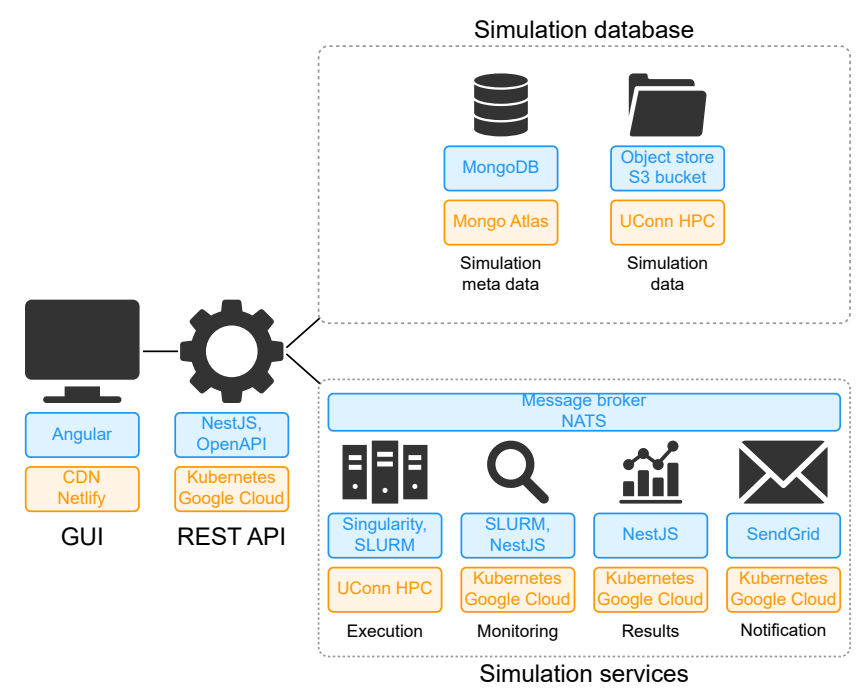

Figure 3. Overview of the implementation (blue) and deployment (orange) of runBioSimulations. The application consists of a GUI; services for executing, monitoring, and logging simulations; and a database of simulations and their results which interact via a REST API. To support multiple simultaneous users, runBioSimulations is deployed using the cloud and HPC.

215 additional conditions and predictions of a model beyond those ${ }_{216}$ reported by its authors. Furthermore, by providing simple 217 access to multiple simulators, runBioSimulations can help 218 authors and model curators verify that simulation results are 219 reproducible across simulation algorithms and simulators.

\section{${ }_{220}$ Collaboration and peer review}

221 We believe that runBioSimulations is similarly well-suited 222 for sharing simulations with collaborators and reviewers. By 223 helping investigators work with different frameworks and 224 algorithms, runBioSimulations makes it easier for investiga225 tors to contribute to multiple collaborations. In particular, 226 runBioSimulations' simulation URLs make it easy for investi${ }_{227}$ gators to share simulations with collaborators, and they enable 228 peer reviewers to access simulations anonymously.

\section{Comparing simulation tools}

230 Because runBioSimulations can execute the same simula${ }_{231}$ tions with multiple simulation tools, runBioSimulations is 232 also well-suited to assessing the compatibility between tools. ${ }_{233}$ For example, investigators could compare results of the same 234 simulations generated with multiple tools to evaluate the 235 performance of the tools, identify inconsistencies among the 236 tools, or detect potential errors in the tools.

\section{Multiscale modeling education}

${ }_{238}$ Furthermore, we believe that runBioSimulations could be a 239 valuable educational tool. In particular, instructors who use 240 runBioSimulations for assignments involving multiple frame241 works would only need to teach their students a single tool. 242 Instructors could also leverage runBioSimulations' simula243 tion results storage for assignments involving the analysis of 244 results of computationally-expensive simulations. 


\section{${ }_{245}$ DISCUSSION}

246 In summary, runBioSimulations provides a simple GUI 247 for executing a broad range of simulations described 248 using community resources such as SBML and SED-ML. 249 Importantly, the community can extend these simulation capa250 bilities by contributing additional standardized simulation 251 tools to the BioSimulators registry. The runBioSimulations 252 GUI also provides users features for managing their simula253 tions, interactively visualizing their results, and sharing their 254 simulations through persistent URLs. In addition, developers 255 can use runBioSimulations' API to build custom applications 256 for executing simulations and/or analyzing simulation results. 257 Together, we believe runBioSimulations will both help authors 258 of in silico experiments share their simulations and help other 259 investigators reproduce and reuse their studies. Ultimately, 260 we believe runBioSimulations will facilitate collaboration and ${ }_{261}$ foster more comprehensive and more predictive models.

\section{Additional modeling formalisms, algorithms, and formats}

${ }_{263}$ We invite developers to extend runBioSimulations to more 264 simulations by contributing additional standardized simula265 tion tools to BioSimulators. To help developers standardize 266 their tools, BioSimulators provides a Python library for 267 executing COMBINE archives, a test suite for validating 268 simulation tools, several examples, and documentation.

\section{More sophisticated data visualizations}

270 We also aim to expand the visualization features of run271 BioSimulations by using Vega to support a broad range of 272 canonical chart types, as well as custom charts, such as 273 network maps. By capturing how charts can be painted with 274 data, Vega would also enable users to reuse diagrams with 275 multiple models and simulations, furthering our goals of reuse 276 and collaboration.

\section{${ }_{277}$ Online platform for sharing entire simulation projects}

${ }_{278}$ Furthermore, we plan to use the runBioSimulations API to 279 develop an online platform that will help authors create and 280 publish entire simulation studies and provide the community 281 a central place to discover and reuse studies. This platform 282 will layer several additional capabilities on top of runBioSim283 ulations. The platform will enable authors to publish models, 284 simulations, simulation results, and data visualizations of sim285 ulation results. The platform will also help the community 286 create and execute variants of published models and simu287 lations to explore alternative simulation conditions, as well 288 as help the community reuse published data visualizations to 289 examine their results. We anticipate this platform will further 290 bolster model reuse, composition, and collaboration.

\section{Additional modeling and simulation tools}

292 Finally, we aim to help the community use runBioSimula293 tions' API to develop additional tools. For example, model 294 repositories could use runBioSimulations to provide capabili295 ties for executing their models, and model format developers 296 could use runBioSimulations to implement test suites for 297 verifying that simulators correctly support their formats.

\section{AVAILABILITY}

299 The application and API are freely available without regis300 tration at https://run.biosimulations.org along with a tutorial, 301 examples, and documentation. The source code is openly 302 available under the MIT license at https://github.com/biosim 303 ulations/Biosimulations.

\section{ACKNOWLEDGEMENTS}

${ }_{305}$ We thank Arthur Goldberg, Herbert Sauro, James Schaff, ${ }_{306}$ Lucian Smith, and the Center for Reproducible Biomedical 307 Modeling for input and feedback.

\section{${ }_{308}$ FUNDING}

${ }_{309}$ This work was supported by National Institutes of Health 310 award P41EB023912.

\section{${ }_{311}$ Conflict of interest statement.}

312 None declared.

\section{${ }_{13}$ REFERENCES}

1. Karr,J. R., Sanghvi,J. C., Macklin,D. N., Gutschow,M. V., Jacobs,J. M., Bolival Jr,B., Assad-Garcia,N., Glass,J. I. and Covert,M. W. (2012) A whole-cell computational model predicts phenotype from genotype. Cell, 150, 389-401.

2. Szigeti,B., Roth,Y. D., Sekar,J. A., Goldberg,A. P., Pochiraju,S. C. and Karr,J. R. (2018) A blueprint for human whole-cell modeling. Curr. Opin. Syst. Biol., 7, 8-15.

3. Faeder,J. R., Blinov,M. L. and Hlavacek,W. S. (2009) Rule-based modeling of biochemical systems with BioNetGen. In Systems Biology pp. 113-167 Springer.

4. Clerx,M., Cooling,M. T., Cooper,J., Garny,A., Moyle,K., Nickerson,D. P., Nielsen,P. M. and Sorby,H. (2020) CellML 2.0. J. Integr. Bioinform., 17, 20200021.

5. Gleeson,P., Crook,S., Cannon,R. C., Hines,M. L., Billings,G. O., Farinella,M., Morse,T. M., Davison,A. P., Ray,S., Bhalla,U. S. et al. (2010) NeuroML: a language for describing data driven models of neurons and networks with a high degree of biological detail. PLoS Comput. Biol., 6, e1000815.

6. Keating,S. M., Waltemath,D., König,M., Zhang,F., Dräger,A., Chaouiya,C., Bergmann,F. T., Finney,A., Gillespie,C. S., Helikar,T. et al. (2020) SBML Level 3: an extensible format for the exchange and reuse of biological models. Mol. Syst. Biol., 16, e9110.

7. Olivier,B. G. and Bergmann,F. T. (2018) SBML level 3 package: flux balance constraints version 2. J. Integr. Bioinform., 15, 20170082.

8. Chaouiya,C., Bérenguier,D., Keating,S. M., Naldi,A., Van Iersel,M. P., Rodriguez,N., Dräger,A., Büchel,F., Cokelaer,T., Kowal,B. et al. (2013) SBML qualitative models: a model representation format and infrastructure to foster interactions between qualitative modelling formalisms and tools. BMC Syst. Biol., 7, 1-15.

9. Harris,L. A., Hogg,J. S., Tapia,J.-J., Sekar,J. A., Gupta,S., Korsunsky,I., Arora,A., Barua,D., Sheehan,R. P. and Faeder,J. R. (2016) BioNetGen 2.2: advances in rule-based modeling. Bioinformatics, 32, 3366-3368.

10. Sneddon,M. W., Faeder,J. R. and Emonet,T. (2011) Efficient modeling, simulation and coarse-graining of biological complexity with NFsim. Nat. Methods, 8, 177-183.

11. Moraru,I. I., Schaff,J. C., Slepchenko,B. M., Blinov,M., Morgan,F., Lakshminarayana,A., Gao,F., Li,Y. and Loew,L. M. (2008) Virtual Cell modelling and simulation software environment. IET Syst. Biol., 2, $352-362$.

3 12. Müssel,C., Hopfensitz,M. and Kestler,H. A. (2010) BoolNet-an R package for generation, reconstruction and analysis of Boolean networks. Bioinformatics, 26, 1378-1380.

13. Helikar,T., Kowal,B. and Rogers,J. (2013) A cell simulator platform: The Cell Collective. Clin. Pharmacol. Ther., 93, 393-395. 
358 14. Olivier,B. G., Swat,M. J. and Moné,M. J. (2016) Modeling and simulation tools: from systems biology to systems medicine. Syst. Med., pp. $441-463$.

15. Ebrahim,A., Lerman,J. A., Palsson,B. O. and Hyduke,D. R. (2013) COBRApy: constraints-based reconstruction and analysis for Python. BMC Syst. Biol., 7, 1-6.

16. Bergmann,F. T., Hoops,S., Klahn,B., Kummer,U., Mendes,P., Pahle,J. and Sahle,S. (2017) COPASI and its applications in biotechnology. $J$. Biotechnol., 261, 215-220.

17. Peters,M., Eicher,J. J., van Niekerk,D. D., Waltemath,D. and Snoep,J. L. (2017) The JWS Online simulation database. Bioinformatics, 33, 15891590.

18. Drawert,B., Hellander,A., Bales,B., Banerjee,D., Bellesia,G., Daigle Jr,B. J., Douglas,G., Gu,M., Gupta,A., Hellander,S. et al. (2016) Stochastic simulation service: bridging the gap between the computational expert and the biologist. PLoS Comput. Biol., 12 , e1005220.

5 19. Choi,K., Medley,J. K., König,M., Stocking,K., Smith,L. P., Gu,S. and Sauro,H. M. (2018) tellurium: an extensible python-based modeling environment for systems and synthetic biology. Biosystems, 171, 74-79.

8 20. Garny,A. and Hunter,P. J. (2015) OpenCOR: a modular and interoperable approach to computational biology. Front. Physiol., 6, 26.

80 21. Gleeson,P., Cantarelli,M., Marin,B., Quintana,A., Earnshaw,M., Sadeh,S., Piasini,E., Birgiolas,J., Cannon,R. C., Cayco-Gajic,N. A. et al. (2019) Open Source Brain: a collaborative resource for visualizing, analyzing, simulating, and developing standardized models of neurons and circuits. Neuron, 103, 395-411.

22. Bergmann,F. T., Cooper,J., König,M., Moraru,I., Nickerson,D., Le Novère,N., Olivier,B. G., Sahle,S., Smith,L. and Waltemath,D. (2018) Simulation experiment description markup language (SED-ML) level 1 version 3 (L1V3). J. Integr. Bioinform., 15.

23. Bergmann,F. T., Adams,R., Moodie,S., Cooper,J., Glont,M., Golebiewski,M., Hucka,M., Laibe,C., Miller,A. K., Nickerson,D. P. et al. (2014) COMBINE archive and OMEX format: one file to share all information to reproduce a modeling project. BMC Bioinformatics, 15 $1-9$.

94 24. Malik-Sheriff,R. S., Glont,M., Nguyen,T. V., Tiwari,K., Roberts,M. G., Xavier,A., Vu,M. T., Men,J., Maire,M., Kananathan,S. et al. (2020) BioModels - 15 years of sharing computational models in life science. Nucleic Acids Res., 48, D407-D415.

25. Scharm,M., Wendland,F., Peters,M., Wolfien,M., Theile,T. and Waltemath,D., The CombineArchiveWeb application-A web based tool to handle files associated with modelling results. Technical report, PeerJ PrePrints (2014)

02 26. Satyanarayan,A., Wongsuphasawat,K. and Heer,J. (2014) Declarative 403 interaction design for data visualization. In Proc. 27th Annи. ACM Symp.

$404 \quad$ User Interface Soft. Technol. pp. 669-678. 